\title{
Tratamiento dietético-nutricional de un trabajador con riesgo cardiovascular en el ámbito universitario
}

\author{
Gerardo Martínez-Martínez ${ }^{a}$, Rocio Ortiz-Moncada a,c, \\ y Ana Noreña-Peña $a^{b, c}$
}

DOI: $10.12961 /$ aprl.2019.22.03.3

Recibido: 13 de enero de 2019

Aceptado: 7 de febrero de 2019

\section{RESUMEN}

Se presenta el caso de un profesor de 47 años, no fumador, con riesgo-cardiovascular (RCV) moderado (2\%) según HeartScore: obesidad (Índice-masa-muscular: 32,8kg/m²); índice cintura-cadera: 1,08, tensión-arterial alta: 150/101mmHg, hipercolesterolemia: $223 \mathrm{mg} / \mathrm{dl}$. La anamnesis-alimentaria inicial (2016) muestra consumo: 2100kcal/día (distribuidas en 24\% proteínas, $45 \%$ grasas, 31\% carbohidratos) y, potasio (51\%) y vitamina-D (42\%) bajos; consume 2 bebidas-alcohólicas/semana. El tratamiento dietético consistió en 2500kcal/día

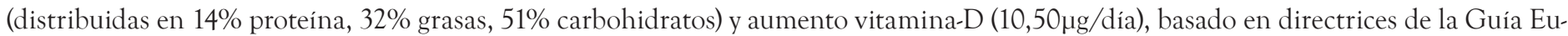
ropea de Prevención Cardiovascular. Después de 1 año de tratamiento se mostraron cambios favorables: Índice-masa-muscular (30,5kg/ $\mathrm{m}^{2}$ ), índice cintura-cadera $(0,98)$, tensión-arterial $(128 / 90 \mathrm{mmHg})$ evidenciados por datos bioquímicos y dietéticos. Este artículo presenta aspectos relevantes de una intervención dietético-nutricional individualizada de factores de RCV en el ámbito laboral y destacan las competencias del dietista-nutricionista y la coordinación del Servicio-Prevención-universitario como activos de salud comunitarios, para revertir parámetros de RCV especialmente antropométricos y tensión-arterial.

PALABRAS CLAVE: factores de riesgo, enfermedades cardiovasculares, tratamiento nutricional, salud laboral, servicios de salud del trabajador, promoción de la salud.

\section{NUTRITIONAL TREATMENT FOR CARDIOVASCULAR RISK IN A UNIVERSITY WORKER}

\begin{abstract}
We present the case of a 47 year old nonsmoking university professor with moderate cardiovascular risk (CVR) (2\%) according to HeartS. core: obesity (body mass index, $32.8 \mathrm{~kg} / \mathrm{m}^{2}$ ); waist-to-hip ratio:1.08; high blood pressure, 150/101 mmHg; and hypercholesterolemia: $223 \mathrm{mg} /$ dL). The initial food inventory (2016) showed a consumption pattern of $2100 \mathrm{kcal} /$ day ( $24 \%$ proteins, $45 \%$ fats, $31 \%$ carbohydrates); low intake of potassium (51\%) and vitamin-D (42\%); consumption of two alcoholic drinks/ per week. Dietary treatment consisted of $2500 \mathrm{kcal} /$ day (distributed as $14 \%$ protein, $32 \%$ fat, $51 \%$ carbohydrates), and increased vitamin D intake $(10.50 \mu \mathrm{g} /$ day), following the European Cardiovascular Prevention Guide. Favorable changes were apparent after one year of treatment: body mass index $\left(30.5 \mathrm{~kg} / \mathrm{m}^{2}\right)$, waist-to-hip ratio $(0.98)$, blood pressure $(128 / 90 \mathrm{mmHg})$, supported by laboratory and dietary data. This article presents relevant aspects of an individualized nutritional intervention targeting CVR factors in the workplace. It highlights the competencies of the dietitian and the coordination of the universitybased occupational health service as community health assets, to revert CVR parameters, especially anthropometric and blood pressure data.
\end{abstract}

KEY WORDS: risk factors, cardiovascular diseases, nutritional therapy, occupational health, ocuppational health service, health promotion.

\section{INTRODUCCIÓN}

Las enfermedades cardiovasculares causaron 17,9 millones de muertes en 2016 en los países desarrollados y éstas pueden pre-

\footnotetext{
a. Departamento de Enfermería Comunitaria, Medicina Preventiva y Salud Pública e Historia de la Ciencia, Universidad de Alicante,

Alicante, España.

b. Departamento de Enfermería, Universidad de Alicante, Alicante, España.

c. Grupo de Investigación en Alimentación y Nutrición (ALINUT),

Alicante, España.
}

Arch Prev Riesgos Labor 2019; 22 (3): $129-133$ venirse atendiendo factores de riesgo cardiovascular $(\mathrm{FRCV})^{1}$. El $35,3 \%$ de trabajadores españoles presentan riesgo cardiovascular (RCV) alto, con tres o más factores de riesgo ${ }^{2}$. En 2015, se registraron $43,5 \%$ eventos mortales (infartos y derrames cerebrales) en el ámbito laboral ${ }^{3}$. La Organización Mundial de la Salud promueve crear entornos saludables en el lugar de trabajo, por los

Autor de correspondencia:

Rocío Ortiz-Moncada

Departamento de Enfermería Comunitaria, Medicina Preventiva,

Salud Pública e Historia de la Ciencia. Universidad de Alicante.

Campus de Sant Vicent del Raspeig. Ap. 99. E-03080 Alacant.

Telf: +34965903962. E-mail: rocio.ortiz@ua.es 
beneficios del estilo de vida saludable: menor absentismo, mayor productividad y mejoras en salud ${ }^{4}$.

La Universidad de Alicante en España, cuenta con un Servicio de Prevención de riesgos laborales (Servicio-Prevención) y un Gabinete de Alimentación y Nutrición (ALINUA) desde 2013 atendido por dietistas-nutricionistas quienes aplican medidas y recursos para controlar enfermedades relacionadas con la dieta y garantizar la prestación de servicios dietéticos ${ }^{5}$. Así, el Servicio-Prevención deriva a ALINUA los casos de usuarios que requieren tratamiento dietético-nutricional y, ALINUA deriva la atención médica-clínica al Servicio-Prevención. La coordinación de dietistas-nutricionistas con los servicios de prevención en España es importante en la resolución de problemas nutricionales en el entorno laboral ${ }^{6}$.

El tratamiento y consejo nutricional en un entorno laboral reporta mejoras en el RCV de los usuarios como consecuencia del manejo dietético de los $\mathrm{FRCV}^{7}$. Sin embargo, la prevención en población activa resulta escasa, a pesar de conocer sus efectos beneficiosos y evidenciar elevadas tasas de mortalidad $^{8}$.

El objetivo del trabajo es describir la intervención dietéticonutricional de un trabajador universitario con FRCV en un entorno laboral favorecedor para su seguimiento.

\section{DESCRIPCIÓN DEL CASO}

Se presenta el caso de un varón de 47 años, geógrafo de profesión, cuya actividad es elaborar informes institucionales complementado con docencia-universitaria (jornada $8 \mathrm{~h} /$ semana). Fue seleccionado a partir de un estudio previo con trabajadores ${ }^{9}$, siendo los criterios de selección: formar parte del personal docenteinvestigador y presentar más de un FRCV.

La información inicial obtenida en la primera visita [21/01/2016] presenta: obesidad I [índice de masa-corporal 32,8kg/ $\mathrm{m}^{2}$ (peso: $112,5 \mathrm{~kg}$; talla: $\left.\left.185 \mathrm{~cm}\right)\right]$, tensión-arterial $(150 / 90 \mathrm{mmHg})(\mathrm{Ta}-$ bla 1). Según la herramienta HeartScore, su RCV a 10 años es moderado (2\%). La anamnesis-alimentaria inicial mediante registro 24 horas (R-24h) indica bajo consumo de frutas (2 raciones/día), legumbres (1 ración/semana) y frutos-secos (consumo nulo). El análisis dietético muestra: $2100 \mathrm{kcal} /$ día (distribución calórica: $24 \%$ proteínas, $45 \%$ grasas, $31 \%$ carbohidratos), un consumo bajo de: potasio (51\%) y vitamina-D (42\%). Se repitieron dos R-24h (fin de semana y entre semana) con resultados similares. Para comprobar posible deficiencia de vitamina-D se solicita analítica sanguínea específica al Servicio-Prevención de la UA. La analítica [21/03/2016] evidencia dislipemia (colesterol-total: >200mg/dL; colesterol-LDL: >100mg/ $\mathrm{dL})$, y se confirma déficit de vitamina-D ( $<30 \mathrm{nmol} / \mathrm{L})$ (Tabla 2).

Se hallaron los siguientes antecedentes familiares en líneamaterna: diabetes mellitus-II, hipercolesterolemia, hipertensión y sobrepeso. Antecedentes personales: sin tratamiento farmacológico pautado, no-fuma, consume cerveza/vino (2 veces/semana) y practica natación 2 horas/semana.

El tratamiento dietético-nutricional se lleva a cabo considerando la Guía Europea de Prevención Cardiovascular? ${ }^{7}$ limitar alcohol (1 copa/semana) aumentando ingesta de agua; aumentar número de ingestas (5 tomas/día). Aumentar consumo de frutas (3 raciones/día), legumbres (2 raciones/semana), alimentos-integrales y vitamina-D mediante lácteos enriquecidos $(10,50 \mu \mathrm{g} /$ día $)$ y exposición solar (3-4 días/semana durante $15 \mathrm{~min} /$ día).

Se realizaron 11 sesiones de seguimiento-dietético durante 1 año (enero-diciembre 2016) con ajustes dietéticos mediante menús, recetas saludables y educación-alimentaria. La tensión-arterial mejoró a las 7 semanas de tratamiento $(125 / 85 \mathrm{mmHg})$. Al final del tratamiento el paciente disminuyó el peso-corporal (7\%) y el índice de cintura-cadera $(0,98)$ (Tabla 1). Los datos bioquímicos señalan mejora de colesterol-HDL y colesterol-LDL después de 4 y 10 meses respectivamente. La vitamina-D experimenta cambios relevantes durante el seguimiento (Tabla 2). Se destaca descenso del indicador de RCV a 10 años en un 1\%. El anexo 1 detalla el análisis-bioquímico de la dieta-inicial (consumo-antes), la dieta-ajustada por tratamiento (consumo-después) y \% adecuación según requerimientosnutricionales. Finalmente indicadores-cualitativos conseguidos aumentando y disminuyendo nutrientes según plan-dietético. Entre los entornos favorecedores del seguimiento-dietético se encuentra la atención personalizada realizada por dietistas-nutricionistas de ALINUA y el apoyo coordinado del Servicio-Prevención.

\section{DISCUSIÓN}

Este artículo presenta aspectos relevantes de éxito de una intervención dietético-nutricional individualizada de FRCV en el ámbito laboral destacando las competencias del dietista-nutricionista y la coordinación con el Servicio-Prevención como activos comunitarios. Estos entornos favorecieron la salud del usuario mediante la disminución peso-corporal, perímetro-cintura, porcentaje-grasa y tensión-arterial.

Los programas de promoción saludables en el entorno laboral fomentan hábitos alimentarios adecuados: mayor consumo de lácteos, frutas, verduras, alimentos integrales y menor consumo de azúcar y refrescos ${ }^{10}$. En este sentido, se hizo hincapié en el consumo de frutas, verduras, legumbres, cereales-integrales y frutos secos por su papel en la reducción del $\mathrm{RCV}^{8}$.

El estudio ICARIA ${ }^{11}$ señala menor discapacidad laboral al mejorar el RCV atribuible al control de la tensión-arterial y de dislipemias. En nuestro caso, la tensión-arterial fue el primer parámetro que mejoró significativamente, reduciendo el RCV del trabajador. La escasez de estudios científicos sobre tratamiento dietético-nutricional en España, supone una limitación para comparar resultados poniendo de manifiesto la necesidad de abrir nuevas líneas de investigación clínica-nutricional. El uso de datos dietéticos autoreportados (anamnesis-alimentaria) es una fortaleza, al ser verificados por diferentes fuentes (bioquímicas, clínicas, antropométricas). Por ello, el tratamiento dietético-nutricional individualizado es eficaz en la mejora de los FRCV. Además, incluir un servicio de nutrición en el lugar de trabajo es una estrategia de salud pública, un activo de salud institucional para fomentar hábitos-alimentarios saludables y de atención personalizada. Finalmente, las políticas de prevención de riesgos-laborales deberían ampliar sus actividades, favoreciendo su articulación con los servicios sociosanitarios y favoreciendo la adopción de medidas adecuadas a la naturaleza del riesgo, tal como evidencia nuestro estudio. 


\section{TABLA 1}

Estudio de caso-nutricional: Evolución de parámetros antropométricos y tensión arterial, 2016.

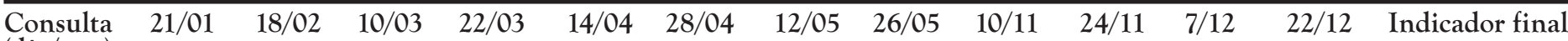
(día/mes)

\section{Indicadores}

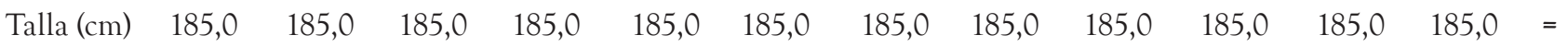

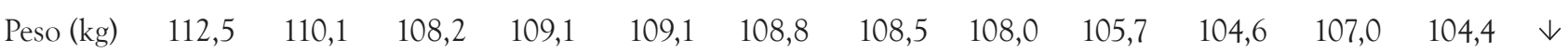

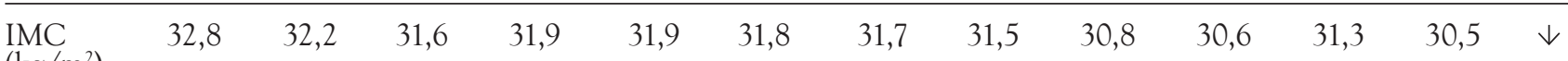

$\left(\mathrm{kg} / \mathrm{m}^{2}\right)$

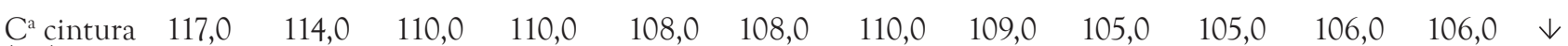

(cm)

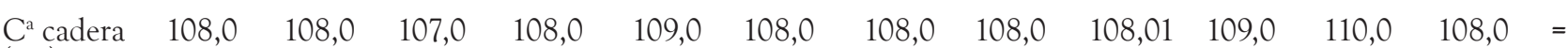
(cm)

\begin{tabular}{|c|c|c|c|c|c|c|c|c|c|c|c|c|c|}
\hline $\mathrm{ICC}^{\mathrm{b}}$ & 1,08 & 10,06 & 1,04 & 1,01 & 0,99 & 1,00 & 1,01 & 1,00 & 0,97 & 0,96 & 0,96 & 0,98 & $\downarrow$ \\
\hline $\begin{array}{l}\text { Grasa } \\
\text { corporal (\%) }\end{array}$ & ${ }_{)^{c}}^{33,4}$ & 33,8 & 34,3 & 32,4 & 33,0 & 32,7 & 33,3 & 33,0 & 31,6 & 34,1 & 33,2 & 32,9 & $\downarrow$ \\
\hline $\begin{array}{l}\text { Grasa } \\
\text { visceralc }^{c}\end{array}$ & 16,0 & 16,0 & 16,0 & 15,0 & 16,0 & 15,0 & 16,0 & 16,0 & 15,0 & 16,0 & 16,0 & 15,0 & $\downarrow$ \\
\hline $\begin{array}{l}\text { Masa } \\
\text { muscular (k }\end{array}$ & ge) & 72,9 & 71,1 & 73,8 & 73,1 & 73,2 & 72,4 & 72,8 & 72,3 & 69,0 & 71,5 & 70,1 & $\downarrow$ \\
\hline $\begin{array}{l}\text { Peso } \\
\text { grasa }(\mathrm{kg})^{\mathrm{c}}\end{array}$ & 37,4 & 37,2 & 37,1 & 35,4 & 36,0 & 35,6 & 36,0 & 35,8 & 33,4 & 35,6 & 35,5 & 34,4 & $\downarrow$ \\
\hline $\begin{array}{l}\text { Peso } \\
\text { agua }(\mathrm{kg})\end{array}$ & 54,7 & 53,4 & 52,1 & 54,0 & 53,5 & 53,6 & 53,0 & 53,3 & 52,9 & 50,4 & 52,3 & 51,3 & $\downarrow$ \\
\hline $\begin{array}{l}\text { Tensión } \\
\text { sistólica }\end{array}$ & 150,0 & 137,0 & 130,0 & 125,0 & 132,0 & 128,0 & 130,0 & 128,0 & 133,0 & 130,0 & 132,0 & 128,0 & $\downarrow$ \\
\hline $\begin{array}{l}\text { Tensión } \\
\text { diastólica }\end{array}$ & 101,0 & 90,0 & 86,0 & 85,0 & 87,0 & 90,0 & 87,0 & 89,0 & 95,0 & 92,0 & 95,0 & $90,0 \downarrow$ & \\
\hline
\end{tabular}

${ }^{\text {a }} \mathrm{C}=$ circunferencia.

${ }^{\mathrm{b}} \mathrm{ICC}=$ índice cintura-cadera.

${ }^{\mathrm{c}}$ indicadores estimados por bioimpedancia eléctrica.

\section{TABLA 2}

Estudio de caso-nutricional: Datos bioquímicos reportados antes y después de la intervención nutricional, 2016.

Meses de tratamiento

Antes
(inicio)

4 meses después

6 meses después

7 meses después

Valor

de refencia

Indicador

Indicadores bioquímicos plasmáticos

\begin{tabular}{|c|c|c|c|c|c|c|}
\hline Glucosa basal (mg/dL) & 101,0 & 98,0 & 92,0 & 90,0 & $60-110 \mathrm{mg} / \mathrm{dL}$ & $\downarrow$ \\
\hline Ácido úrico (mg/dL) & 6,2 & 7,3 & 7,4 & 7,0 & $2,5-7,5 \mathrm{mg} / \mathrm{dL}$ & $\uparrow$ \\
\hline Creatinina (mg/dL) & 1,00 & 1,30 & 1,23 & 1,09 & $<1,30 \mathrm{mg} / \mathrm{dL}$ & $\uparrow$ \\
\hline Colesterol total $(\mathrm{mg} / \mathrm{dL})$ & 223,0 & 231,0 & 198,0 & 229,0 & $<200 \mathrm{mg} / \mathrm{dL}$ & $\uparrow$ \\
\hline Triglicéridos (mg/dL) & 145,0 & 135,0 & 128,0 & 131,0 & $<200 \mathrm{mg} / \mathrm{dL}$ & $\downarrow$ \\
\hline HDL-colesterol (mg/dL) & 42,0 & 60,0 & 44,0 & 47,0 & $>40 \mathrm{mg} / \mathrm{dL}$ & $\uparrow$ \\
\hline LDL-colesterol (mg/dL) & 152,0 & 144,0 & 128,0 & 156,0 & $<100 \mathrm{mg} / \mathrm{dL}$ & $\uparrow$ \\
\hline Potasio $(\mathrm{mmol} / \mathrm{L})$ & 4,7 & 4,5 & 5,0 & 5,2 & $3,5-5,1 \mathrm{mmol} / \mathrm{L}$ & $\uparrow$ \\
\hline Vitamina D (ng/mL) & 26,9 & 24,0 & 30,8 & 25,4 & $30-150 \mathrm{ng} / \mathrm{mL}$ & $\downarrow$ \\
\hline
\end{tabular}


ANEXO 1

Estudio de caso: Análisis bioquímico de la dieta antes y después del tratamiento.

\begin{tabular}{|c|c|c|c|c|c|c|}
\hline Nutrientes & $\begin{array}{l}\text { Consumo } \\
\text { antes } \\
21-01-2016\end{array}$ & $\begin{array}{l}\text { Consumo } \\
\text { después } \\
30-01-2016\end{array}$ & $\begin{array}{l}\text { Requerimientos } \\
\text { nutricionales }\end{array}$ & $\begin{array}{l}\% \text { de adecuación } \\
\text { dieta antes }(100 \%)\end{array}$ & $\begin{array}{l}\text { \% de adecuación } \\
\text { dieta después }(100 \%)\end{array}$ & $\begin{array}{l}\text { Indicador } \\
\text { cualitativo }\end{array}$ \\
\hline Energía (kcal) & 2100,0 & 2460,0 & 2900,0 & 72,4 & 84,8 & $\uparrow$ \\
\hline Proteínas (g) & 92,5 & 94,0 & 108,7 & 85,0 & 86,4 & $\uparrow$ \\
\hline Lípidos (g) & 105,0 & 90,0 & 96,6 & 108,7 & 93,1 & $\downarrow$ \\
\hline $\mathrm{AGS}^{\mathrm{a}}(\mathrm{g})$ & 36,0 & 16,0 & 24,1 & 149,3 & 66,3 & $\downarrow$ \\
\hline $\mathrm{AGM}^{\mathrm{b}}(\mathrm{g})$ & 48,0 & 50,0 & 48,3 & 99,3 & 103,5 & $\uparrow$ \\
\hline $\operatorname{AGP}^{c}(g)$ & 12,0 & 19,0 & 24,1 & 49,7 & 78,8 & $\uparrow$ \\
\hline $\mathrm{HC}^{\mathrm{d}}$ total $(\mathrm{g})$ & 164,0 & 318,0 & 398,0 & 41,1 & 79,7 & $\uparrow$ \\
\hline Azúcares (g) & 48,5 & 120,0 & & & & $\uparrow$ \\
\hline Fibra (g) & 17,0 & 47,0 & 45,0 & 37,7 & 104,4 & $\uparrow$ \\
\hline Sodio (mg) & 2760,0 & 1950,0 & 1500,0 & 184,0 & 130,0 & $\downarrow$ \\
\hline Potasio (mg) & 2400,0 & 4860,0 & 4705,0 & 51,0 & 103,0 & $\uparrow$ \\
\hline Calcio (mg) & 1100,0 & 810,0 & 800,0 & 137,0 & 101,0 & $\downarrow$ \\
\hline Magnesio (mg) & 654,0 & 490,0 & 350,0 & 184,0 & 140,0 & $\downarrow$ \\
\hline Fósforo (mg) & 1450,0 & 1590,0 & 700,0 & 207,0 & 225,0 & $\uparrow$ \\
\hline Hierro (mg) & 10,0 & 19,0 & 10,0 & 100,0 & 190,0 & $\uparrow$ \\
\hline Zinc (mg) & 10,0 & 12,0 & 15,0 & 66,0 & 80,0 & $\uparrow$ \\
\hline Vit. A (ug) & 601,5 & 1600,0 & 1000,0 & 60,0 & 160,0 & $\uparrow$ \\
\hline Vit. D (ug) & 601,5 & 1600,0 & 1000,0 & 60,0 & 160,0 & $\uparrow$ \\
\hline Vit. E (mg) & 8,5 & 20,0 & 12,0 & 70,0 & 165,0 & $\uparrow$ \\
\hline Tiamina (mg) & 0,95 & 1,70 & 1,10 & 84,0 & 154,0 & $\uparrow$ \\
\hline Riboflavina (mg) & 1,50 & 1,85 & 1,70 & 88,0 & 108,0 & $\uparrow$ \\
\hline Niacina (mg) & 22,0 & 36,0 & 19,0 & 115,0 & 189,0 & $\uparrow$ \\
\hline Vit. B6 (mg) & 1,4 & 3,2 & 1,8 & 78,0 & 178,0 & $\uparrow$ \\
\hline Ácido fólico (mg) & 260,5 & 506,0 & 200,0 & 130,0 & 253,0 & $\uparrow$ \\
\hline Vit. B12 (ug) & 4,1 & 11,5 & 2,0 & 205,0 & 575,0 & $\uparrow$ \\
\hline Vit. C (ug) & 51,0 & 127,5 & 60,0 & 85,0 & 212,0 & $\uparrow$ \\
\hline
\end{tabular}

a. $\mathrm{AGS}=$ ácidos grasos saturados .

b. AGM= ácidos grasos monoinsaturados.

c. $\mathrm{AGP}=$ ácidos grasos poliinsaturados.

d. $\mathrm{HC}=$ hidratos de carbono.

\section{AGRADECIMIENTOS}

Agradecemos especialmente al paciente por su participación y compromiso en el tratamiento dietético. A los profesionales del equipo del Gabinete de Alimentación y Nutrición de la Universidad de Alicante (ALINUA), por las orientaciones técnicocientíficas durante el tratamiento. Al Servicio de Prevención de la Universidad de Alicante por su coordinación en el proceso de seguimiento médico-clínico del paciente, como a la Facultad de Ciencias de la Salud. 


\section{BIBLIOGRAFÍA}

1. World Health Organization. Cardiovascular diseases (CVDs). Key facts. [Actualizado 17 may 2017; citado 11 jul 2017]. Disponible en: https://www.who.int/ news-room/fact-sheets/detail/cardiovascular-diseases-(cvds).

2. Instituto Nacional de Seguridad e Higiene en el Trabajo (INSHT). Factores de riesgo de enfermedades cardiovasculares en la población trabajadora según la Encuesta Nacional de Salud 2011/2012. [Publicado nov 2015; citado 11 jul 2017]. Disponible en: http://www.oect.es/Observatorio/5\%20Estudios\%20 tecnicos/Riesgos\%20especificos/Riesgo\%20cardiovascular/Ficheros\%20e\%20 informes/Riesgo\%20cardiovascular.pdf.

3. Ministerio de Empleo y Seguridad Social. Estadísticas de Accidentes de Trabajo. [Publicado 2016; citado 11 jul 2017]. Disponible en: http://www.mitramiss. gob.es/estadisticas/eat/eat16/Resumen_resultados_ATR_2016.pdf.

4. Organización Mundial de la Salud. Salud de los trabajadores: plan de acción mundial. 60a Asamblea mundial de la salud. Ginebra, Suiza. [Publicado 23 may 2007; citado 18 nov 2018]: Disponible en: https://www.who.int/occupational_ health/WHO_health_assembly_sp_web.pdf.

5. Davó MC, Gil-González D, Vives-Cases C, Alvarez-Dardet C, Ronda E, OrtizMoncada R, et al. ¿Quiénes y qué pueden hacer en salud pública? Las competencias profesionales como base para la elaboración de programas en el Espacio Europeo de Educación Superior. Gac Sanit. 2009; 23: 5-12.

6. Baladia E, Basulto J. Grupo de Revisión y Posicionamiento de la Asociación España de Dietistas-Nutricionistas (GREP/AED-N). El dietista-nutricionista en atención primaria de salud. Actividad Dietética. 2007; 34: 24-38.

7. Gambin L, Rueda JC, Jimenez D, Bastida M, Fernández AM, Rodríguez J. Influencia de la implantación de un servicio de nutrición en el control de factores de riesgo cardiovascular en una población trabajadora. Revista de la Asociación Española de Especialistas en Medicina del Trabajo. 2017; 26: 178-85.

8. Piepoli MF, Hoes AW, Agewall S, Albus C, Brotons C, Catapano AL, et al. European Guidelines on cardiovascular disease prevention in clinical practice: The Sixth Joint Task Force of the European Society of Cardiology and Other Societies on Cardiovascular Disease Prevention in Clinical Practice (constituted by representatives of 10 societies and by invited experts) Developed with the special contribution of the European Association for Cardiovascular Prevention \& Rehabilitation (EACPR). Eur Heart J. 2016; 37: 2315-81.

9. Norte AI, Sansano M, Martínez JM, Sospedra I, Hurtado JA, Ortiz R. Estudio de factores de riesgo de enfermedad cardiovascular en trabajadores universitarios españoles. Nutr Hosp. 2016; 33: 644-48.

10. Paredes G, Ruiz L, González N. Healthy habits and nutritional status in the work environment. Revista Chilena de Nutrición. 2018; 45:119-12.

11. Calvo-Bonacho E, Catalina-Romero C, Cabrera M, Fernández-Labandera C, Sánchez MA, Brotons C, et al. Asociación entre la mejora en el perfil de riesgo cardiovascular y los cambios en la incapacidad temporal: resultados del estudio ICARIA. Rev Esp Cardiol. 2017; 70: 941-51. 